\title{
Alkoholkonsum von Jugendlichen als Praxis der Raumaneignung am Beispiel der Stadt Zürich
}

\author{
Sara Landolt, Norman Backhaus, Zürich
}

\section{Einleitung}

Ende Januar 2008 gaben die Schweizerischen Bundesbahnen (SBB) bekannt, dass ab April 2008 in ihren Bahnhöfen ab 22 Uhr kein Alkohol mehr über die Gasse verkauft wird (Ladengeschäfte und Kioske). Davon erhoffen sich die SBB «mehr Sauberkeit und Ordnung in den Bahnhöfen» (SBB 2008). In der Begründung heisst es weiter:

«(...), dass sich zur späten Stunde namentlich auch Jugendliche in ganzen Gruppen in den Bahnhöfen Alkohol in grossen Mengen beschaffen und konsumieren» (SBB 2008).

Ein Bericht auf der Titelseite der Quartierzeitung ZüRICH NoRD beginnt mit der Einleitung:

«Wenn am Wochenende am Schaffhauserplatz leere Flaschen herumlagen, stammten sie meist aus dem Coop [Lebensmittelgrossverteiler]. Dieser verkauft deshalb 2007 gar keinen Alkohol an Minderjährige» (STUDER 2007: 1).

Von den herumliegenden Flaschen auf dem Schaffhauserplatz bis zum Verzicht von Coop, in dieser einen Filiale keinen Alkohol mehr an unter 18-Jährige zu verkaufen - obwohl der gesetzlich geregelte Verkauf von alkoholischen Getränken an Kinder und Jugendliche in der Schweiz für Bier und Wein bei 16 Jahren liegt (SR 680) - fanden Aushandlungen von Normen und Regeln statt, die in dieser Öffentlichkeit gelten (sollen). Sie wurden ausgelöst durch den Alkoholkonsum und die damit verbundenen Handlungen Jugendlicher im Quartier sowie durch involvierte verschiedene andere Akteure: Quartierverein, Coop, Gemeinwesenarbeiter, Jugendarbeiter, Jugendliche, um nur einige $\mathrm{zu}$ nennen. Diese Beispiele sollen die momentane öffentliche Debatte über Alkoholkonsum illustrieren, bei welcher oft Jugendliche im Zentrum stehen. Sie kann durch zwei dominante Diskursstränge charakterisiert werden: Entweder wird (präventiv-)medizinisch die Zunahme des binge drinking (Rauschtrinken) unter Jugendlichen thematisiert, oder es wird fehlende «Sauberkeit und Ordnung» mit dem Alkoholkonsum von Jugendlichen ausserhalb des privaten Raums verbunden. Während bei beiden Argumentationslinien Forderungen oder die Umsetzung von Massnahmen mit räumlichen, zeitlichen oder ans Alter gebundenen Restriktionen verbunden werden, beinhaltet die zweite eine Auseinandersetzung über Werte, Normen und Regeln, die an bestimmten Orten gelten (sollen).
Im Gegensatz zu dieser öffentlich geführten Debatte mangelt es an wissenschaftlichen Studien, die Alkoholkonsum von Jugendlichen mit Fragen der Raumaneignung verbinden. Während der Alkoholkonsum als wichtige Komponente von Jugendkulturen (z.B. Chatterton \& Hollands 2003; Miles 2000) sowie bei der Konstruktion von Männlichkeit und Weiblichkeit (Gough \& Edwards 1998; KoliP 2002) erkannt wurde, ist die Verbindung von Alkoholkonsum und städtischem Leben nur am Rande Gegenstand von Forschungen (JAYNE et al. 2006).

In diesem Artikel wird im Kontext der skizzierten öffentlichen Debatte der Fokus auf die in Verbindung mit Alkoholkonsum stattfindenden Raumaneignungen Jugendlicher gerichtet. Dabei stellen sich folgende Fragen: Wo trinken Jugendliche Alkohol? Gibt es Konflikte mit anderen Nutzungen? Auf einer abstrakteren Ebene ist dabei von Interesse, welche Räume durch die Ortswahl und den Alkoholkonsum konstruiert werden, welche Regeln in diesen «Trinkräumen» gelten (werden dabei soziale Differenzen (re)produziert?), und inwiefern sich Jugendliche durch diese Praxis der Raumaneignung an einer Neuaushandlung von Regeln an «öffentlichen Orten» beteiligen. Bevor Forschungsmethoden und Resultate präsentiert werden, soll die Forschung in den Kontext der jugendlichen Raumaneignung eingebettet werden.

\section{Raumaneignung, Regionalisierung und Transgression}

Aus bisheriger geographischer Forschung zu Raumnutzungen und -aneignungen Jugendlicher sind va. die Restriktionen, die Erwachsene Jugendlichen bei der Raumnutzung setzen, und die daraus resultierende marginale Stellung der Jugendlichen in Städten bekannt (z.B. VALENTINE et al. 1998). Öffentlicher Raum, der im Fokus dieser Studien steht, und der oft diffus als «alles ausserhalb des Zuhauses» konzeptualisiert ist, wird dementsprechend als produced as adult space bezeichnet, in dem Jugendliche mit ihren räumlichen Praktiken - wie etwa dem Herumhängen in Einkaufspassagen (NARIN et al. 2003) - schnell auf Ablehnung durch Erwachsene stossen. Jugendliche sind zwar von den primären Herstellungsprozessen öffentlicher Räume - Planung und Gestaltung - grösstenteils ausgeschlossen, doch gelingt es ihnen teilweise, bei der Inbesitznahme dieser Räume ihre Bedürfnisse, die nicht mit den beabsichtigten Raumaneignungen übereinzustim- 
men brauchen, ein- oder zumindest anzubringen. Der Raumaneignung wohnt dabei sowohl das Moment des Schaffens eines eigenen Raums (mit eigenen Werten Normen, Regeln, Strukturen) inne, der losgelöst von bestehenden Räumen existiert, als auch das Markieren einer Gegenwelt zur Welt der Erwachsenen (vgl. Matthews et al. 1998; ReutLinger 2003). Neben der Gegenüberstellung der Kategorien Jugendliche und Erwachsene fokussiert die neueste Forschung auf die Analyse von weiteren Differenzen und Ungleichheiten wie z.B. Geschlecht, soziale Schicht, Ethnizität oder Lebensstil (z.B. Karsten \& Pel 2000). So zeigte etwa Thomas (2005) am Beispiel Charleston (USA), dass afroamerikanische Mädchen beim Herumhängen in öffentlichen Räumen deutlich andere Erfahrungen mit der Polizei machen als gleichaltrige weisse Mädchen. Dies bedeutet, dass Raumaneignungen durch Jugendliche differenziert betrachtet werden müssen.

Hier wird der Raum als soziale Konstruktion verstanden, die durch alltägliches Handeln permanent produziert und reproduziert wird (BACKHAUS \& MüLLER 2006; WerLen 1997). Die dabei erfolgende Zuschreibung von Bedeutungen geschieht über Raumnutzung - bzw. physische Raumaneignung - sowie über die mentale Auseinandersetzung mit einem Raum (vgl. BACKHAUS \& MülLER 2006). Man spricht bei diesem Prozess, bei dem alltägliche Routinen eine wichtige Rolle spielen, auch von Regionalisierung (vgl. GIDDENS 1996; WERLEN 1997). Mit den Bedeutungszuschreibungen werden Regeln und Normen mit materiellen Artefakten verbunden, welche bestimmte Nutzungen ermöglichen und andere einschränken können. Orte werden zu normativen Räumen, denen zugeschrieben wird, was an einem Ort angebracht ist und was nicht: Was ist in place, was ist out of place (Cresswell 1996). Die Verbindung von Orten mit den ihnen angemessenen Verhaltensweisen wird oft erst durch nicht angebrachte Handlungen sichtbar; Cresswell (1996) nennt diese transgressiv. Diesen ist eigen, dass sie eine Reaktion auslösen, die enthüllt, was bisher als korrekt und natürlich angesehen wurde. Diese Reaktion ist ein Indikator, dass eine Grenze zwischen «normal» und «anormal» überschritten wurde (CRESSWEll 1996). Alkoholkonsum von Jugendlichen bei Bahnhöfen, auf öffentlichen Plätzen und vor Läden ist eine Praxis, die - bedenkt man die eingangs dargestellte öffentliche Debatte - als transgressiv verstanden werden kann. Es ist deshalb angebracht, bei diesen Handlungen von Transgression zu sprechen, da sie sich, im Unterschied zum Widerstand, nicht über die Intention, sondern über das Resultat der Handlung definiert (Cresswell 1996). Das heisst, es geht nicht darum, was die Jugendlichen mit dem Trinken bezwecken wollen, sondern darum, wie darauf reagiert wird; ferner, welche Bedeutung der Handlung erstens im Nachhinein und zweitens nicht nur von den Jugendlichen selbst, sondern von der gesamten Gesellschaft beigemessen wird. Da die Praxis des Alkoholkonsums jedoch nicht an diesem Punkt abbricht, sondern - quasi in einem veränderten gesellschaftlichen Kontext - weitergeht, werden in der Diskussion folgende Fragen aufgenommen: Sind sich die Jugendlichen mit der Zeit bewusst, dass ihren Handlungen etwas Herausforderndes anhaftet? Kann davon ausgegangen werden, dass sie daher bewusst - im Sinne eines Widerstandes - Alkohol out of place konsumieren?

Raumaneignungen können zu Konflikten führen. Dies kann einerseits durch eine offene Konfrontation mit anderen Interessen in situ stattfinden, wenn verschiedene Formen und Bedürfnisse der Raumaneignung aufeinanderprallen. Die Konflikte können aber auch verdeckt sein, wenn ein Ort aufgrund von Handlungen und Nutzungen der einen für andere negativ belegt wird. Dabei hat sich die Bedeutung des Raumes für diese Gruppe verändert. Aus einem zuvor positiv konnotierten Raumausschnitt kann z.B. ein so genannter Angstraum werden, der im Extremfall fortan von dieser Gruppe gemieden wird (ausführlicher dazu vgl. SCHELLER 1995).

\section{Methodisches Vorgehen}

Das empirische Material für diesen Artikel bilden Beobachtungen und acht Gruppengespräche mit weiblichen und männlichen Jugendlichen. Die Gespräche wurden in gleichgeschlechtlichen Freundespaaren und -gruppen (2-4 Personen) durchgeführt. Alle Interviewten wohnen in Zürich, sind zwischen 15 und 17 Jahre alt, unterscheiden sich jedoch bezüglich weiterer sozialer Strukturkategorien voneinander. Sie besuchen unterschiedliche Schulstufen, haben eine Lehre begonnen oder befinden sich auf Lehrstellensuche. Ihre Familien können sowohl unterschiedlichen Schichten zugeordnet werden, wie auch unterschiedlichen Herkunftskulturen. Die Auswahl der Untersuchungsteilnehmer basiert auf einem theoretischen sampling (nach STRAUSS \& CORBIN 1996), wobei die Strukturkategorien Geschlecht, Bildung und kulturelle Zugehörigkeit sowie das Freizeitverhalten berücksichtigt wurden. Die Jugendlichen wurden über Schulen, Jugendräume, die Vermittlung von Sozialarbeitern und über direktes Ansprechen in öffentlichen Räumen gefunden. Alle Gespräche wurden aufgenommen, transkribiert und anschliessend mittels theoretischen Kodierens nach Strauss \& CORBIN (1996) analysiert.

\section{Orte des Alkoholkonsums von Jugendlichen}

Ein Merkmal des Alkoholkonsums von Jugendlichen ist, dass neben traditionellen Orten des Alkoholkonsums - z.B. zu Hause, in Wirtshäusern, Klubs - an 
Orten getrunken wird, die nicht primär mit Alkoholkonsum verbunden werden. So trinken Jugendliche auch im Freien, wo sie den Abend verbringen, treffen sich und trinken vor Läden, die Alkohol verkaufen, oder trinken den billig gekauften Alkohol vor den Klubs, die sie anschliessend besuchen (vgl. ChatTerTON \& Hollands 2003 für England). Auch suchen sie Orte auf, die ausserhalb der direkten elterlichen Kontrolle liegen. Dies geschieht speziell dann, wenn sie einen von den Eltern (vermeintlich) nicht tolerierten Alkoholkonsum pflegen, wie die folgenden Ausschnitte einerseits aus einem Gespräch mit Eva und ihrer Freundin, anderseits mit einer Gruppe befreundeter männlicher Jugendlicher um Erkan zeigen (bei allen verwendeten Namen handelt es sich nicht um die tatsächlichen Namen der Jugendlichen).

Eva: «Mit meinen Eltern trinke ich schon ab und zu ein Glas Wein, wenn man aber im Ausgang trinkt, dann trinkt man mehr als ein Glas und das würde ich jetzt nicht mit meinen Eltern machen. (...) nur ein Glas, weil mehr, das wäre mir peinlich».

Interviewerin: «Bist du, sind deine Eltern, seid ihr Muslime?»

Erkan: «Ja. Und da würde man eigentlich auch nicht trinken. Aber mein Vater trinkt selber. Also, er sagt einfach: 'Wenn du besoffen nach Hause kommst, dann ist's nicht mehr schön für dich.> (...) Er findet's nicht gut. Und auch die Religion. Beides irgendwie. Und äh, wegen dem Ruf, was die andern noch erzählen und so. Es wäre eine Blamage für die ganze Verwandtschaft».

Die im ersten Ausschnitt erwähnte Peinlichkeit bezieht sich - wie sich im Verlaufe des Gespräches mit Eva und Tina zeigte - auf das Überschreiten des aus der Sicht ihrer Eltern «normalen» Alkoholkonsumverhaltens für Jugendliche. Bei Erkan erscheinen, zusätzlich zu den Erwartungen und der damit verbundenen möglichen Enttäuschung der Eltern, die Blamage für die Familie sowie das Überschreiten einer religiösen Vorschrift als erklärende Faktoren.

Im Folgenden wird einerseits anhand von Eva und Tina, zwei 17-jährigen Mädchen aus der Oberschicht, anderseits anhand einer Gruppe männlicher Unterschichtsjugendlicher um Erkan exemplarisch gezeigt, wie unterschiedlich die Orte sein können, an denen Alkohol konsumiert wird. Darauf basierend wird diskutiert, ob und wo diese Praxis out of place ist und durch die Ortswahl des Alkoholkonsums eine Transgression darstellt, die hegemoniale Regeln und Normen in Frage stellt.

\subsection{Nutzung bestehender Trinkorte vs. Produktion von neuen Trinkräumen}

Tina: «Mein Geld, ja, für Klubs, da geht meines hin. Wir sind ja eben am [XY], das ist ja eine Privatschule und die mei- sten bekommen Geld wie nichts von ihren Eltern, und die haben wirklich einfach immer viel Geld (...). So ein Abend unterwegs ist teuer. Die Klubs, Eintritt, die Getränke, das Taxi nach Hause. Die, die Geld haben, kaufen den Champagner. So eine Flasche kostet 400 Franken».

Interviewerin: «Weshalb gehst du da gerne hin?» Tina: «[R] ist ein cooler Ort. (...) Da ist nicht einfach jeder. (...) Da hat's Leute so wie wir. Da hat man eigentlich auch seine Ruhe. (...) Die, die nerven, sind nicht dort».

Interviewerin: «Die, die nerven?»

Tina: «Ja die halt, die dich so anmachen». (Im Folgenden geht die Befragte detailliert auf diese «Anmache» ein).

Erkan: «Wenn wir gestresst sind, saufen oder kiffen wir. Saufen macht Spass, ist Fest haben. Sagt einer von uns, lass uns heute Freinacht machen, dann machen wir. Kaufen Wodka, suchen sicheren Platz, manchmal am See, manchmal an anderem Ort, da oben im Wald. Letztes Mal sind wir auf Mädchenjagd am Hauptbahnhof gegangen, saufen, gehen zu einem andern Platz, laufen auf der Strasse, manchmal mit Bus, wenn wir laufen und die Polizei kommt, sagen wir, wir sind eingeschlafen, haben beim Freund zu Hause TV geschaut. Manchmal machen die dann einen Alkoholtest, müssen wir Ausweis zeigen, notieren unsere Namen, kein Problem. Dann sagen sie: «Geht nach Hause Jungs», manchmal nehmen sie uns auch auf den Polizeiposten mit».

Erkan und Tina trinken an völlig unterschiedlichen Orten Alkohol. Während Tina mit Freunden und Freundinnen teure Klubs besucht, kennt Erkan die Regeln der Strasse, um draussen mit seinen Freunden Alkohol trinken zu können.

Der Einlass in die Welt der teuren Klubs verlangt von Jugendlichen grosse finanzielle Ressourcen (Eintrittspreis und hohe Konsumationspreise). Dies ist jedoch nicht die einzige Restriktion. Neben den finanziellen Ressourcen erwähnen die Jugendlichen immer wieder die Alterslimite von 18, die sie mit gefälschten Ausweisen oder solchen älterer Geschwister umgehen, und den zum Klub passenden Habitus als weitere Einlasshürden, die beide durch Türsteher kontrolliert werden. Chatterton und Hollands (2003: 56) beschreiben in ihrer Untersuchung zur Produktion, Regulation und Konsumption städtischer Nachträume in England einen Wandel der Funktion der Türsteher, die zusätzlich zum hard man neu auch style selectors seien. Dies ist eine Bezeichnung, die auch die Erzählungen der Jugendlichen dieser Untersuchung treffend wiedergibt. So meinte Tina etwa, dass die Türsteher schon auch schauen würden, ob man zum Klub passt. Diese Restriktionen führen dazu, dass exklusive Trinkräume nicht von jedermann besucht werden und in diesen eine relative Homogenität bezüglich Schicht und Habitus der Besuchenden herrscht. Gerade Mäd- 
chen betonen diese Homogenität besonders und in positiver Art. In ihrer eigenen sozialen Schicht fühlen sie sich im Umgang mit dem anderen Geschlecht sicherer, Kontakte spielen sich innerhalb geteilter Normen und Regeln ab, die ihnen vertrauter sind und gegenseitig eingehalten und akzeptiert werden. Am Beispiel des Flirtens wird die Norm des gegenseitigen Respektierens sichtbar.

Tina beschreibt dies so: «Wir sprechen miteinander, sie bieten dir was zum Trinken an. Man trinkt, tanzt».

Diese Beschreibung kommt ohne wertende Erklärungen aus. Im Kontrast dazu steht die Beschreibung der Mädchen zur Art wie sie von habitusfernen Jugendlichen, die sie im Gespräch als «aus dem Balkan kommend» bezeichnen, angesprochen werden. Diese hätten ein «aufdringliches Verhalten», was die Befragten dann inhaltlich ausführten.

Sowohl die Auswahl von anerkannten Trinkorten als auch der Heimweg per Taxi unterstützen das Ausbleiben von Konflikten in der Öffentlichkeit, wenn Tina und ihre Freunde und Freundinnen Alkohol konsumieren. Die Kapitalien (finanzielles Kapital, passende Kleidung, gefälschte Ausweise), die Tina und Eva zur Verfügung haben, ermöglichen ihnen Raumaneignungen, die anderen, die nicht über diese Kapitalien verfügen, vorenthalten bleiben. Das Fortführen dieser Praxis führt zur Stabilisierung des dominanten Images dieser Orte.

Andere Orte suchen der 15-jährige Erkan und seine Freunde - die sich «FX Fighters» (der Name wurde geändert) nennen - aus, wenn sie Alkohol trinken. Mit der Aussage «einen sicheren Platz suchen» fällt auch schon das erste Kriterium auf, welches von einem Ort erfüllt werden muss, damit dieser zu einem Trinkraum werden kann. Erkan und seine Freunde betonen im Gespräch mehrmals, dass sie einen Ort zuerst «auschecken» müssen, um abschätzen zu können, ob er «Fluchtwege» bereithält. So unterschiedlich die von ihnen aufgesuchten Orte zu sein scheinen - vom sehr belebten Hauptbahnhof über die weit über die Stadt hinaus beliebten Seeanlagen zu Kinderspielplätzen im Wohnquartier der «FX Fighters» und am Quartierrand gelegenen Wald - haben alle miteinander gemein, dass sie für den Fall einer Störung über Fluchtwege verfügen. Im Gegensatz zu den oben genannten exklusiven Trinkorten handelt es sich ausschliesslich um Orte, bei denen die Nutzung als Trinkräume nicht ursprünglich vorgesehen war, die also bei der Planung und Gestaltung nicht darauf ausgelegt wurden, (auch) Trinkorte Jugendlicher zu sein. So werden sie erst in dem Moment, in welchem die Jugendlichen da auch wirklich Alkohol trinken, zu Trinkräumen. Das Trinken kann dabei allein schon deshalb Aufmerksamkeit erregen, weil an «falschen Orten» oder, mit CRESSWELL (1996) ausgedrückt, out of place getrunken wird, während Tinas und Evas Trinkpraxis durch die Ortswahl in place ist. Dadurch, dass die «FX Fighters» an unterschiedlichen Orten - allesamt nicht anerkannte Trinkorte - Alkohol trinken, zeigt sich jedoch anhand der unterschiedlichen Reaktionen auf diese Praxis, resp. des Ausbleibens von Reaktionen an Orten wie dem Trinken im Wald, dass das Trinken ausserhalb der Trinkorte nicht per se out of place ist.

\subsection{Aushandlungen von Regeln und Normen}

Aus dem individuellen «kleinen» Überschreiten der elterlichen Regeln wird eine sichtbare Transgression gesellschaftlicher Normen, wenn Orte als Trinkorte ausgewählt werden, deren sonstige Nutzung und Bedeutungsbelegung damit im Konflikt stehen. Die mit dem Trinken verbundene Überschreitung von Normen und Regeln bezieht sich meist auf Sauberkeit und Ordnung. Dass Normen überschritten werden, wird durch die Reaktion der Gesellschaft ersichtlich: zum Beispiel durch Anzeigen von Anwohnenden wegen nächtlicher Ruhestörung, Medienberichte, zeitliche Einschränkungen des Alkoholverkaufs, wie in den SBB-Bahnhofläden, und Gesetzesänderungen zur Einschränkung des Alkoholkonsums auf öffentlichem Grund, wie dies beispielsweise kürzlich in Chur geschah (Verwaltung Chur, Zugriff 25.02.2008). Durch die Wahl eines Trinkortes und die damit verbundene Diskussion, was an einem Ort möglich ist und was nicht, wird das «private» Trinken zum bewusst provozierten oder unbewusst in Kauf genommenen Aushandeln von in der Öffentlichkeit gültigen Regeln. In welche Richtung diese Auseinandersetzungen gehen, z.B. ob dieses Verhalten öffentliche Diskussionen auslöst, die einen restriktiveren Umgang mit Alkohol verlangen - wie in den Eingangsbeispielen dargestellt - ist in diesem Moment nebensächlich. Im Zentrum steht das Sichtbarmachen des eigenen Bedürfnisses der Jugendlichen nach Räumen, in denen sie sich aufhalten können. So wurden in den Gruppengesprächen mehrmals fehlende Räume, eine prinzipielle Ablehnung jugendlichen Verhaltens in öffentlichen Räumen sowie ungenügende Öffnungszeiten der Jugendräume reklamiert. Die Aussagen einiger Jugendlicher, sie hätten ein Recht, im öffentlichen Raum zu sein, unterstreicht dies. Damit demonstrieren die Jugendlichen auch, dass sie eine gerne übergangene gesellschaftliche Minderheit mit eingeschränkten Rechten sind.

Wie der Aushandlungsprozess ausgestaltet wird, hängt nicht nur von der Praxis Jugendlicher ab, sondern auch davon, wie die Behörden darauf reagieren. Die Stadt Zürich schreibt sich das Paradigma einer Koexistenz im öffentlichen Raum auf die Fahne. Das heisst, dass weder auf einen Rückzug der Jugendlichen hingearbeitet wird, noch dass sich die Gemeinwesen- und 
Sozialarbeit anwaltschaftlich hinter sie stellt. Vielmehr sollen - wie im aktuellen Legislaturschwerpunkt «Jugend in Zürich: FreiRäume, Arbeit und Sicherheit» zu lesen ist - «Nutzungskonflikte (...) offen und innovativ in Form von Aushandlungsprozessen angegangen [werden]» (STADT ZüRICH 2006: 20).

Aushandlungsprozesse geschehen jedoch nicht immer so sichtbar und in einem durch die Behörden moderierten Kontext. Sie finden auch auf individueller und familiärer Ebene und in subtilerer Form statt, und nicht immer führen sie zu Lösungen, die von allen Beteiligten akzeptiert werden. Erkan, der sich davor hütet, betrunken nach Hause zu kommen, erzählt später, wie ihn seine Eltern manchmal nach alkoholreichen Freinächten von der Polizeistation abholen müssen. Das Trinken draussen endet nicht nur damit, dass er nun doch betrunken nach Hause kommt, sondern beinhaltet zusätzlich, dass ihn die Eltern auf der Polizeiwache abholen müssen. Dabei kommt es zu Vermischungen von Öffentlichem und Privatem, und die von ihm geschaffene Regionalisierung bricht zusammen. Erkan, der im Gruppengespräch zuerst heroisch über seine Freinächte in dem von ihnen geschaffenen Raum berichtet, der stolz mitteilt, dass er es ist, der Kontakt zu einem Ladenbesitzer hat, der ihnen Alkohol verkauft, obwohl dies das Jugendschutzgesetz verbietet, wird bei dieser Gesprächspassage zum Jungen, der leise erzählt, wie er darüber nachdachte, weshalb er in diese Situation gekommen war. Was bei der Raumaneignung und Konstruktion von Trinkräumen wichtige Ressourcen sind und Erkan innerhalb der Gruppe hohes soziales Kapital verschafft, kippt mit der durch die Polizeikontrolle ausgelösten Verlagerung ins elterliche und private Umfeld ins Gegenteil.

Erkan: «Vor Kollegen, du bist der Held. Du bist der Beste, bekommst Achtung, wenn du Scheiss machst, wenn Polizei wegen dir kommt und du auf Polizeistation musst. $\mathrm{Zu}$ Hause du schlägst Kopf an die Wand und fragst dich, weshalb habe ich das gemacht».

Erkans Raumaneignung, die Konstruktion von Trinkräumen, die an konkreten Orten verortet ist, wirkt für andere auch, wenn er und seine Freunde nicht mehr präsent sind.

Interviewerin: «Was du da jetzt beschrieben hast, bedeutet das auch, dass du deswegen gewisse Orte meidest?»

Eda: «Also wegen den Jungs? Nein, also es gibt schon Plätze, [dort bei XY], da gehe ich am Abend nicht durch. Da sitzen sie, trinken und kiffen».

Jana: «Da müssen wir auch nicht hin. Wir wissen schon wo».

Für Eda und Jana haben die erwähnten Plätze eine andere Bedeutung erhalten, sie regionalisieren sie als Angsträume oder zumindest als unangenehme Räume. Indem sie dort nicht mehr hingehen, betreiben sie selbst eine Art negative Raumaneignung oder Raumenteignung resp. nehmen an der von den trinkenden Jungen «vorgegebenen» Regionalisierung teil. An diesem Beispiel sieht man, dass Rauman- bzw. -enteignungen nicht etwas sind, das von bestimmten Gruppen alleine durchgesetzt wird. Was hier geschieht, ist ein gegenseitiger Prozess, der, ausgelöst durch eine trinkende Gruppe, zu neuen Regionalisierungen führt, die weitgehend in den Köpfen der anderen Beteiligten abläuft und für Eda und Jana zur Routine wird, indem sie diese Orte systematisch - das heisst hier v.a. nachts - meiden. Es werden jedoch nicht alle Trinkorte zu Angsträumen aus Sicht der Mädchen. Ihre Aufzählung der Orte, welche sie meiden und ihre Geschichten zu Orten, die sie gerade auch um Jungen zu treffen aufsuchen (z.B. der Hauptbahnhof), lassen auf Folgendes schliessen: Je kontrollierter ein Trinkort ist (beim Hauptbahnhof erwähnen sie etwa die Bahnpolizei, die den Raum für sie sicherer und angenehmer macht), desto eher wird dieser nicht zum Angstraum für Mädchen.

\section{Fazit}

Alkoholkonsum von Jugendlichen kann aus einer Perspektive betrachtet werden, die diesen Konsum als Praxis der Raumaneignung untersucht. Durch Alkoholkonsum in Abhängigkeit des Ortes werden

1. Räumen neue Bedeutungen zugeschrieben (z.B. im Fall des Konsums im Wald).

2. Gleichzeitig erfolgt eine moralische und räumliche Grenzüberschreitung, die bisherige Normalitäten sichtbar macht und herausfordert. Dies stellt für verschiedene Akteure eine Bedeutungsverschiebung und Veränderung der Handlungsmöglichkeiten und der weiteren Aneignungen dar (z.B. im Fall der Seeanlagen oder des Hauptbahnhofs).

3. Es wird im Sinne bestehender Bedeutungszuweisungen gehandelt, die dadurch reproduziert werden (z.B. im Fall von Klubbesuchen).

Die Wahl der Trinkorte ist somit entscheidend, ob der Alkoholkonsum zu Konflikten und Auseinandersetzungen führt. Da bei Trinkorten mit dem geringsten Konfliktpotential Eintrittskapitalien verlangt werden, die von den Unterschichtsjugendlichen nicht erbracht werden können, trinken einerseits diese meist an konfliktreicheren Orten, anderseits werden gewisse soziale Differenzen reproduziert. So bewirkt die Raumaneignung der Jungen im Fall 1) und 2), dass sich Mädchen an diesen Orten unwohl fühlen und diese meiden. Die Mädchen finden sich in der klassischen Rolle der Verletzlichen, der in der Raumaneignung Schwächeren und greifen mit dem 
Meiden gewisser Orte zu bestimmten Zeiten zur Strategie des Rückzugs. Sie überlassen das Feld den Jungen.

Im Fall 2) beinhaltet die Raumaneignung eine Transgression, die zu Aushandlungsprozessen von in der Öffentlichkeit gültigen Regeln führt. Die Stadt Zürich hat sich zum Ziel gesetzt hat, dass

«Jugendliche und junge Erwachsene (...) genügend geeignete Freiräume in Gebäuden und Plätzen im öffentlichen

Raum finden, wo sie nach eigenen Vorstellungen präsent und aktiv sein [können]» (STADT ZÜRICH 2006: 20).

Dadurch vertritt sie bei den Aushandlungsprozessen weder einen strikt anwaltschaftlichen Ansatz gegenüber der Klientel der Jugendlichen, noch verfolgt sie einen repressiven Ansatz mit räumlichen oder zeitlichen Verboten. Sie ordnet auch nicht die «störenden» Bedürfnisse der Jugendlichen den «hegemonialen» Bedürfnissen nach Ruhe und Ordnung unter. Dieses Paradigma eröffnet Raum zur gemeinsamen Auseinandersetzung: Personen, die sich durch Jugendliche gestört fühlen, sollten nicht nur die Polizei oder Gemeinwesenarbeiter rufen. Jugendliche wiederum sollten ihrerseits nicht nur in Form der Raumaneignung an diesen Aushandlungen teilnehmen. Vielmehr wäre es wünschenswert, dass ein Austausch mittels eines «runden Tisches» erfolgt. Dadurch kann auf ein gegenseitiges Verstehen der unterschiedlichen Raumzuschreibungen hingearbeitet werden (MATHOW et al. 2008).

\section{Literatur}

Backhaus, N. \& U. Müller (2006): Regionalisierung: eine konstruktivistische Perspektive. - In: BACKHAUs, N. \& U. Müller-BöKer (Hrsg.): Gesellschaft und Raum - Konzepte und Kategorien. - = Schriftenreihe Humangeographie 22, Zürich: Geographisches Institut der Universität Zürich: 13-29.

Chatterton, P. \& R. Hollands (2003): Urban nightscapes. Youth culture, pleasure spaces and corporate power. - London: Routledge.

Cresswell, T. (1996): In place/out of place. Geography, ideology, and transgression. - Minneapolis, London: University of Minnesota Press.

Giddens, A. (1996): Konsequenzen der Moderne. - Frankfurt am Main: Suhrkamp.

Gough, B. \& G. EdwARds (1998): The beer talking: four ladies, a carry out and the reproduction of masculinities. - In: Sociological Review 46, 3: 409-435.

JAYNe, M., Holloway, S.L. \& G. VAlentine (2006): Drunk and disorderly: alcohol, urban life and public space. - In: Progress in Human Geography 30, 4: 451-468.

Karsten, L. \& E. Pel (2000): Skateboarders exploring urban public space: ollies, obstacles and conflicts. - In: Journal of Housing and the Built Environment 15: 327-340.
KoLIP, P. (2002): Geschlechtsspezifisches Risikoverhalten im Jugendalter: empirische Befunde und theoretische Erklärungsansätze. - In: Bundesgesundheitsblatt - Gesundheitsforschung - Gesundheitsschutz 45: 885 888.

Mathow, C., Schubiger, B., Holler, C. \& R. DellsPERGER (2008): Nutzungskonflikte im öffentlichen Raum: kreative Handlungsansätze mit Jugendlichen. - Stadt Zürich, Sozialdepartement (internes Arbeitspapier).

Matthews, H., Limb, M. \& B. Percy-Smith (1998): Changing worlds: the microgeographies of young teenagers. - In: Tijdschrift voor Economische en Sociale Geografie 89, 2: 193-202.

Miles, S. (2000): Youth lifestyles in a changing world. - Buckingham: Open University Press.

Nairn, K., Panelli, R. \& J. McCormack (2003): Destabilizing dualisms: young people's experiences of rural and urban environments. - In: Childhood 10, 1: 9-42.

Reutlinger, C. (2003): Jugend, Stadt und Raum. Sozialgeographische Grundlagen einer Sozialpädagogik des Jugendalters. - Opladen: Leske + Budrich.

SBB Medienmitteilung (2008): Bahnhofläden verzichten auf Alkoholverkauf ab 22.00 Uhr. - http:// mct.sbb.ch/mct/konzern_dienstleistungen/konzern_ medien/ konzern_medienmitteilungen 29.01.2008.

SCHELlER, A. (1995): FRAU MACHT RAUM: geschlechtsspezifische Regionalisieurngen der Alltagswelt als Ausdruck von Machtstrukturen. - = Schriftenreihe Anthropogeographie 16, Zürich: Geographisches Institut der Universität Zürich.

SR 680: Bundesgesetz über die gebrannten Wasser (Alkoholgesetz) vom 21.6.1932 (Stand am 1.1.2008). - Bern.

STADT ZÜRICH (Hrsg.) (2006): Legislaturschwerpunkt 2006-2010. - Zürich.

Strauss, A. \& J. Corbin (1996): Grounded Theory. Grundlagen qualitativer Sozialforschung. - Weinheim: Beltz.

STUdER, R. (2007): Alkohol gibt es nur noch für Erwachsene. - In: Zürich Nord v. 11.1.2007, Zürich: 1.

Thomas, M. (2005): Girls, consumption space and the contradictions of hanging out in the city. - In: Social \& Cultural Geography 6, 4: 587-605.

Valentine, G., Skelton, T. \& D. Chambers (1998): Cool places: an introduction to youth and youth cultures. - In: Skelton, T. \& G. Valentine (Hrsg.): Cool places: geographies of youth cultures. - London, New York: Routledge: 1-34.

Verwaltung Chur (2008): Urnenbotschaft zur Volksabstimmung vom 24. Februar 2008. - http://www.chur. ch/d/search/index.cfm 25.02.2008.

WERLEN, B. (1997): Sozialgeographie alltäglicher Regionalisierungen 2: Globalisierung, Region und Regionalisierung. - = Erdkundliches Wissen 119, Stuttgart: Franz Steiner Verlag. 
Zusammenfassung: Alkoholkonsum von Jugendlichen als Praxis der Raumaneignung am Beispiel der Stadt Zürich

Vor dem Hintergrund öffentlicher Debatten, die Alkoholkonsum von Jugendlichen mit fehlender «Sauberkeit und Ordnung» im öffentlichen Raum verbinden und Restriktionen fordern, werden aus einer konstruktivistischen Perspektive die in Verbindung mit Alkoholkonsum stattfindenden Raumaneignungen Jugendlicher untersucht. Die empirische Basis bilden Gruppengespräche mit Jugendlichen. Es zeigt sich, dass Jugendliche unterschiedlicher Schichten an verschiedenen Orten Trinkräume mit je eigenen Normen und Regeln konstruieren. In Abhängigkeit von den Orten bereits zugeschriebenen Bedeutungen wird die Praxis des Alkoholkonsums von Jugendlichen zur transgressiven Handlung, die hegemoniale Normen sichtbar macht und herausfordert. Durch diese Praxis können z.B. auch die so konstruierten Trinkräume der einen zu Angsträumen für andere werden. Durch die Wahl eines Trinkortes und die damit verbundenen Diskussionen, was an einem Ort möglich ist und was nicht, führt das «private» Trinken unter Jugendlichen zum Aushandeln von in der Öffentlichkeit gültigen Regeln. Am Beispiel der Stadt Zürich wird gezeigt, dass die Ausgestaltung dieses Prozesses auch davon abhängt, wie Behörden darauf reagieren.

Schlüsselwörter: Raumaneignung, Jugendliche, Alkohol, qualitative Sozialforschung, Nutzungskonflikte

\section{Résumé: La consommation d'alcool des jeunes comme pratique de territorialisation: I'exemple de la ville de Zurich}

En adoptant une perspective constructiviste, cet article étudie les pratiques de territorialisation liées à la consommation d'alcool des jeunes, au regard des débats actuels qui associent cette consommation d'alcool juvénile à des atteintes à «la propreté et à l'ordre» de l'espace public. La base empirique de cette étude est constituée d'entretiens de groupe avec des jeunes. Elle révèle que, selon la couche sociale dont ils viennent, les jeunes se construisent des espaces de consommation d'alcool différents ayant chacun leurs normes et leurs règles propres. En fonction des significations déjà rattachées à un lieu, la pratique de consommation d'alcool juvénile devient une pratique transgressive, qui rend visible les normes hégémoniques et les met à l'épreuve. L'article montre ensuite comment les espaces de boisson des uns peuvent devenir des espaces de peur pour d'autres. A travers le choix d'un lieu pour boire et la négociation de ce qu'il est possible d'y faire ou non, la consommation d'alcool «privée» des jeunes devient un acte de négociation des règles valables dans l'espace public. L'exemple de la ville de Zurich montre que le déroulement de ces processus dépend aussi de la manière dont les autorités réagissent.

Mots-clés: appropriation de l'espace, jeunesse, alcool, méthode qualitative, conflit d'usage

\begin{abstract}
Spatial appropriation through alcohol consumption - teenage drinking in the City of Zurich Media discourse in Switzerland on teenage alcohol consumption impairing «cleanliness and order» in public spaces is increasing. Calls are being made in articles and political debates to curb these «activities». The focus of this article is on spatial appropriations linked to alcohol consumption by teenagers from a constructivist point of view. Group discussions with youths serve as the empirical basis. The results indicate that teenagers with different backgrounds use and create different kinds of drinking spaces with specific rules and norms. Depending on the meanings previously attributed to these spaces, the practice of teenage drinking becomes transgressive whilst concurrently making hegemonic norms visible by challenging them. This can lead to these drinking spaces becoming spaces of fear for others. In drinking space selection and subsequent discussions about what is possible in a particular place and what not, «private» drinking practices among teenagers become negotiations about acceptable rules and norms in public spaces. Experiences made by the City of Zurich's authorities indicate that the form of this negotiation process also depends on how the authorities react to teenage drinking practices in public spaces.
\end{abstract}

Keywords: appropriation of space, youth, alcohol, qualitative methods, conflict of interest

Dipl.-Geogr. Sara Landolt, PD Dr. Norman Backhaus, Geographisches Institut der Universität Zürich, Winterthurerstrasse 190, CH-8057 Zürich, Schweiz. e-mail:

sara.landolt@geo.uzh.ch

norman.backhaus@geo.uzh.ch

Manuskripteingang/received/manuscrit entré le

3.11.2008

Annahme zum Druck/accepted for publication/accepté pour l'impression: 8.9.2009 